Research Square
Preprints are preliminary reports that have not undergone peer review.

They should not be considered conclusive, used to inform clinical practice, or referenced by the media as validated information.

\title{
Cross-Sectional Associations Between 24-hour Activity Behaviours and Motor Competence in Youth: A Compositional Data Analysis
}

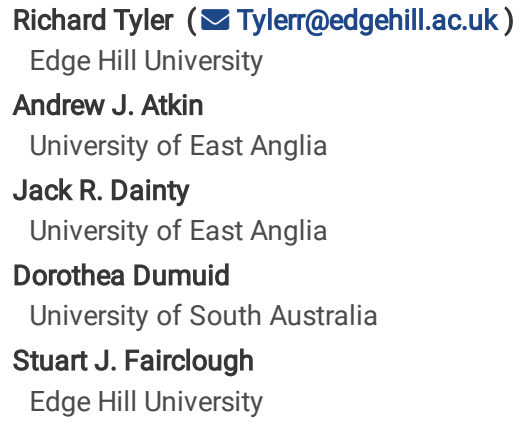




\section{Abstract \\ Background}

The study aimed to examine the cross-sectional associations between 24-hour activity compositions and motor competence in children and adolescents, while stratifying by sex and school type, and investigate the predicted differences in motor competence when time was reallocated between activity behaviours.

\section{Methods}

Data were collected from 359 participants (aged 11.5 \pm 1.4 years; $49.3 \%$ boys; $96.9 \%$ White British). Seven-day 24-hour activity behaviours (sleep, sedentary time, light physical activity (LPA), moderate-to-vigorous physical activity (MVPA)) were assessed using wrist-worn accelerometers. Motor competence outcomes were obtained using the Dragon Challenge (process, product, time, and overall scores). Linear mixed models examined associations between activity behaviour compositions and motor competence outcomes for all participants and stratified by school type (primary or secondary) and sex. Post-hoc analyses modelled the influence of reallocating fixed durations of time between activity behaviours on outcomes.

\section{Results}

In all participants, relative to other activity behaviours, MVPA had the strongest associations with motor competence outcomes. The stratified models displayed that MVPA had the strongest associations with outcomes in both sexes, irrespective of school type. The largest positive, and negative predicted differences occurred when MVPA replaced LPA or sleep, and when LPA or sleep replaced MVPA, respectively.

\section{Conclusions}

Relative to other activity behaviours, MVPA appears to have the greatest influence overall on motor competence outcomes. Reallocating time from LPA or sleep to MVPA reflected the largest positive predicted changes in motor competence outcomes. Therefore, our findings reinforce the key role of MVPA for children's and adolescents' motor competence.

\section{Practical Implications:}

- This study provides empirical evidence that more MVPA, relative to other activity behaviours, is associated with positive motor competence outcomes.

- Our findings reinforce the key role of MVPA for children and adolescents' motor competence and highlight the collective influence of activity behaviours on motor competence outcomes, which could guide the focus of motor competence interventions.

- Promoting and protecting opportunities for MVPA is beneficial for motor competence in all children and adolescents and practical approaches to increase MVPA are advocated.

\section{Background:}

Childhood and adolescence are critical periods and provide a window of opportunity for the development of motor competence(1). Motor competence is an umbrella term used to represent an individual's ability to perform skilfully on a wide range of motor tasks(1-5). The term encapsulates fundamental, combined, and complex movement skills, which are amalgamated to form general, refined, and specific movement patterns, and utilised to perform goaldirected movements/motor tasks in various physical activity (PA) and sporting pursuits(1-6). Therefore, it is purported that motor competence is an important precursor for increasing positive health trajectories, particularly PA, across the lifespan $(4,5,7,8)$.

Previous studies and systematic reviews have reported evidence for positive associations between motor competence and PA levels(2, 7), and that a positive feedback loop or reciprocal relationship exists, in which youth with greater levels of PA, develop better motor competence, leading to further increases in PA engagement $(2,5,7,8)$. However, a recent systematic review investigated the bi-directional association between PA and motor competence and found no evidence for the pathway from more PA to better motor competence(3). The authors suggest that, among other reasons, the common placement of devicemeasured (i.e., accelerometer) PA at the waist, may not capture the intensity of discrete skilled movements, such as throwing, meaning that the association between PA and motor competence will likely be low(3). Therefore, there is a need to investigate associations between device-measured PA and motor competence using accelerometry worn at alternative locations, such as the wrist. An additional reason for the lack of association between PA and motor competence may be that previous research has focused on time allocated to moderate-to-vigorous physical activity (MVPA) and its association to motor competence in isolation, or only with partial adjustment for time in other physical behaviours (i.e., sedentary time (ST), light physical activity (LPA), and sleep) $(2,3,7)$. Accounting for the inter-relations of MVPA, LPA, ST, and sleep are important because these activity behaviours are constrained to the 24-hours of the day. Thus, a change in one behaviour results in change in others (e.g., increase in ST could reflect a decrease in MVPA)(9), and therefore the association between motor competence and a specific behaviour also depends on other behaviours.

More recently, studies using compositional data analyses and compositional isotemporal substitution have investigated associations between activity behaviour compositions and motor competence; specifically, fundamental movement skill proficiency, in pre-school and primary school-aged children(10-13), without stratifying by sex. The stratification of sex may be important given the sex differences in the preference of physical activity types engaged in 
throughout childhood and adolescence, and that these different activity types could have diverging roles in developing motor competence7. In addition, none of these compositional studies have investigated motor competence using proficiency in combinations of fundamental, combined and complex movement skills in children and adolescents. Previous studies on this topic have used primarily process-based assessments of motor competence(10-13). Given that individual process- and product-based assessments shine a light on different and limited aspects of motor competence(1), hybrid-based motor competence assessments (using both a process- and product-based approach) have been developed for children and adolescents $(1,6)$. Thus, investigating associations between activity behaviour compositions and motor competence using a measure that utilises a hybrid-based approach to provide a more holistic view of motor competence, is warranted.

Reflecting on this, we aimed to (i) examine the cross-sectional associations between 24-hour activity compositions and motor competence (using a hybridbased measure of motor competence) in children and adolescents while stratifying by sex and school type, and (ii) investigate the predicted differences in motor competence when time was reallocated between activity behaviours.

\section{Methods:}

Following institutional ethical approval (\#SPA-REC-2018-007) written informed parent consent and participant assent were obtained from 382 students from ten primary and two secondary schools in the West Lancashire region of northwest England. From these, 359 students ( 23 were absent on data collection days) took part in the study between April 2019 and March 2020, as part of a wider project described elsewhere(14).

Participants' ethnicity, dates of birth, and home postal codes were obtained from the schools' information management systems and used to calculate decimal age and 2019 Indices of Multiple Deprivation (IMD) deciles(15). Height and body mass were measured using a portable stadiometer [Seca 213, Seca Ltd, Birmingham, UK] and calibrated scales [Seca 813, Seca Ltd, Birmingham, UK], respectively. From these, body mass index (BMI) and BMI z-scores were calculated, and age- and sex-specific BMI cut-points were used to classify weight status(16).

To obtain activity behaviours, participants wore triaxial accelerometers [ActiGraph GT9X, ActiGraph, Pensacola, FL, USA], set at 100Hz, on the non-dominant wrist for $24 \mathrm{~h} \cdot$ day $^{-1}$ over 7 days. Data were processed in R [http://cran.r-project.org] using GGIR [v1.9.0](17), which performed auto-calibration procedures, identified non-wear, and converted raw triaxial accelerometer signals into 1 omnidirectional measure of acceleration (Euclidean Norm Minus-One; ENMO)(14, $18,19)$. ENMO values were averaged per $5 \mathrm{~s}$ epoch over the 7 monitored days to represent average acceleration expressed as milligravitational units ( $\mathrm{m} g$ ). Participants were excluded if accelerometer post-calibration error was $>10 \mathrm{mg}$ and if $<3$ valid days of wear (i.e., $\geq 16 \mathrm{~h} \cdot$ day $^{-1}$ ) were recorded(14). Youth-specific non-dominant wrist ENMO cut-points of $50 \mathrm{mg}$ and $200 \mathrm{mg}(20,21)$, defined estimated ST/LPA, and MVPA, respectively. Sleep duration was estimated using a polysomnography-validated accelerometer algorithm(22).

Participants undertook the Dragon Challenge (DC) assessment of motor competence, involving nine tasks (Balance Bench, Core Agility, Wobble Spot, Overarm Throw, Basketball Dribble, Catch, Jumping Patterns, T-Agility, and Sprint) completed in a timed circuit(6). Each DC task requires participants to apply a different combination of fundamental, combined, and complex movement skills(6). Good validity and reliability of the assessment has been established(6). Using standardised methodology(6), scoring was completed in situ, by an expert assessor with >100 hours of DC training/in situ experience (with good intraand inter-rater reliability against other expert assessors, using pre-recorded DC reliability videos (all ICCs $>0.9$ )). The assessor had no prior knowledge of participants' movement capabilities. Participants were assessed against criteria on the process (quality of movement technique) and product (successfully achieving the outcome/goal) for each task, and time taken to complete the circuit (hybrid-based approach)(6). From these, process, product, time, and overall scores were calculated (motor competence outcomes), with larger scores indicative of higher levels of motor competence(6).

Multiple imputation was performed for missing data (24.5\% of activity behaviour data; $0.3 \%$ BMI z-score; $2.5 \%$ IMD decile; $1.1 \%$ DC scores) in IBM SPSS Statistics [v25, IBM Inc., NY, USA], using the expectation maximisation algorithm(14). Compositional analyses were conducted using R v3.6.2 [www.rproject.org](14). Sets of pivot coordinates (time-use compositions expressed as four specific sets of three isometric log ratio (ILR) coordinates) were used as explanatory variables in regression analyses(14). A mixed model approach ('school' as the single, random intercept) analysed the association of DC scores with the time-use ILRs(14), whilst adjusting for age, sex, BMI z-scores, and IMD deciles(10-13). Additional sex- and school-stratified analyses were performed.

Models were checked to ensure assumptions for the use of linear mixed effects were met. The ANOVA table of the model fit displayed whether the set of timeuse ILRs were significantly associated with the selected DC score. If significant, four models were carried out, each using a different set of pivot co-ordinates, so that they examined associations with one activity behaviour, relative to all remaining behaviours. The most dominant behaviour was determined(14), and was the focus of compositional isotemporal substitution analyses to model the influence of reallocating fixed time durations ( $5,10,15,20$ minutes) between it and the other behaviours(9). Effect-sizes (ES) for predicted differences in DC scores were calculated as ratios of the model residual standard deviation(14, 23).

\section{Results:}

Mean and standard deviations of the measured variables and geometric means of activity behaviours (linearly adjusted to equal 1440 minutes) are presented in Table 1. Participants were predominantly White British (96.9\%), of medium-to-high socioeconomic position (IMD decile 7.3 \pm 2.3 ), $49.3 \%$ were boys, and $24.6 \%$ were overweight or obese. There was high compliance to wearing the accelerometers $\left(5.9 \pm 1.6\right.$ days of valid wear for $22.8 \pm 1.0$ hour.day $\left.{ }^{-1}\right)$. Participants spent $44.1 \%$ of the 24 -hour day in ST, $37 \%$ in sleep, $14.6 \%$ in LPA, and $3.5 \%$ in MVPA. Only $23.1 \%$ of participants averaged $\geq 60$ minutes MVPA per day(24) and $72.4 \%$ had $<9$ hours sleep per night(25). Primary school participants spent more time in sleep, LPA, and MVPA, and less in ST than their older peers. Dragon Challenge scores for participants aligned to published national/normative data(6); the participants did not have high levels of motor competence nor particularly low levels. The secondary school participants performed better in DC scores than their younger peers. 
Table 1

Descriptive characteristics of study participants and descriptive accelerometer and motor competence variables (Mean (SD) unless indicated)

\begin{tabular}{|c|c|c|c|c|c|c|c|c|c|}
\hline Variables & $\begin{array}{l}\text { All } \\
\text { schools }\end{array}$ & $\begin{array}{l}\text { Primary } \\
\text { schools } \\
\text { (All) }\end{array}$ & $\begin{array}{l}\text { Secondary } \\
\text { schools } \\
\text { (All) }\end{array}$ & $\begin{array}{l}\text { All } \\
\text { schools } \\
\text { (Girls) }\end{array}$ & $\begin{array}{l}\text { Primary } \\
\text { schools } \\
\text { (Girls) }\end{array}$ & $\begin{array}{l}\text { Secondary } \\
\text { schools } \\
\text { (Girls) }\end{array}$ & $\begin{array}{l}\text { All } \\
\text { schools } \\
\text { (Boys) }\end{array}$ & $\begin{array}{l}\text { Primary } \\
\text { schools } \\
\text { (Boys) }\end{array}$ & $\begin{array}{l}\text { Secondary } \\
\text { schools } \\
\text { (Boys) }\end{array}$ \\
\hline $\mathrm{N}$ & 359 & 210 & 149 & 182 & 103 & 79 & 177 & 107 & 70 \\
\hline Age (years) & $\begin{array}{l}11.5 \\
(1.4)\end{array}$ & $10.4(0.7)$ & $13.0(0.3)$ & $\begin{array}{l}11.5 \\
(1.5)\end{array}$ & $\begin{array}{l}10.3 \\
(0.7)\end{array}$ & $13.1(0.3)$ & $\begin{array}{l}11.5 \\
(1.3)\end{array}$ & $\begin{array}{l}10.5 \\
(0.7)\end{array}$ & $13.0(0.3)$ \\
\hline Girls (\%) & 50.7 & 49.0 & 53.0 & - & - & - & - & - & - \\
\hline \multicolumn{10}{|l|}{ Ethnicity } \\
\hline White British (\%) & 96.9 & 97.1 & 96.6 & 98.9 & 99.0 & 98.7 & 94.9 & 95.3 & 94.3 \\
\hline \multicolumn{10}{|l|}{ Socioeconomic status } \\
\hline IMD score & $\begin{array}{l}13.0 \\
(8.9)\end{array}$ & $11.3(6.5)$ & $15.4(11.0)$ & $\begin{array}{l}13.3 \\
(9.9)\end{array}$ & $\begin{array}{l}11.6 \\
(7.1)\end{array}$ & $15.4(12.4)$ & $\begin{array}{l}12.7 \\
(7.7)\end{array}$ & $\begin{array}{l}11.0 \\
(5.8)\end{array}$ & $15.3(9.3)$ \\
\hline IMD decile & $7.3(2.3)$ & $7.8(2.0)$ & $6.7(2.5)$ & $7.4(2.4)$ & $\begin{array}{l}7.7 \\
(2.1)\end{array}$ & $6.8(2.6)$ & $7.3(2.2)$ & $\begin{array}{l}7.8 \\
(1.9)\end{array}$ & $6.5(2.3)$ \\
\hline Height (cm) & $\begin{array}{l}149.1 \\
(11.3)\end{array}$ & $143.0(8.3)$ & $157.8(9.1)$ & $\begin{array}{l}149.0 \\
(11.7)\end{array}$ & $\begin{array}{l}142.4 \\
(8.6)\end{array}$ & $157.6(9.6)$ & $\begin{array}{l}149.3 \\
(10.9)\end{array}$ & $\begin{array}{l}143.6 \\
(8.0)\end{array}$ & $158.1(8.6)$ \\
\hline Body Mass (kg) & $\begin{array}{l}43.1 \\
(12.2)\end{array}$ & $37.9(9.7)$ & $50.4(11.6)$ & $\begin{array}{l}43.6 \\
(12.9)\end{array}$ & $\begin{array}{l}38.0 \\
(10.4)\end{array}$ & $51.0(12.2)$ & $\begin{array}{l}42.5 \\
(11.5)\end{array}$ & $\begin{array}{l}37.8 \\
(9.2)\end{array}$ & $49.7(10.9)$ \\
\hline $\mathrm{BMI}\left(\mathrm{kg} \cdot \mathrm{m}^{-2}\right)$ & $\begin{array}{l}19.1 \\
(3.6)\end{array}$ & $18.3(3.2)$ & $20.1(3.8)$ & $\begin{array}{l}19.4 \\
(3.9)\end{array}$ & $\begin{array}{l}18.5 \\
(3.4)\end{array}$ & $20.5(4.1)$ & $\begin{array}{l}18.8 \\
(3.3)\end{array}$ & $\begin{array}{l}18.1 \\
(3.0)\end{array}$ & $19.7(3.4)$ \\
\hline BMI z-score & $\begin{array}{l}0.39 \\
(1.21)\end{array}$ & $0.38(1.23)$ & $0.41(1.17)$ & $\begin{array}{l}0.35 \\
(1.23)\end{array}$ & $\begin{array}{l}0.35 \\
(1.24)\end{array}$ & $0.35(1.23)$ & $\begin{array}{l}0.44 \\
(1.18)\end{array}$ & $\begin{array}{l}0.42 \\
(1.22)\end{array}$ & $0.47(1.12)$ \\
\hline \multicolumn{10}{|l|}{ Weight Status } \\
\hline Underweight (\%) & 7.9 & 8.2 & 7.5 & 8.3 & 8.7 & 7.7 & 7.3 & 7.5 & 7.1 \\
\hline Healthy (\%) & 67.6 & 66.7 & 68.9 & 66.9 & 64.1 & 70.5 & 68.4 & 69.2 & 67.1 \\
\hline Overweight (\%) & 19.8 & 20.5 & 18.9 & 18.2 & 20.4 & 15.4 & 21.5 & 20.6 & 22.9 \\
\hline Obese (\%) & 4.7 & 4.8 & 4.7 & 6.6 & 6.8 & 6.4 & 2.8 & 2.8 & 2.9 \\
\hline \multicolumn{10}{|l|}{ Accelerometer $^{a}$} \\
\hline ST (min. day ${ }^{-1}$ ) & 635.8 & 580.6 & 693.6 & 639.6 & 592.8 & 701.0 & 632.7 & 598.9 & 684.8 \\
\hline LPA (min·day $\left.{ }^{-1}\right)$ & 210.1 & 211.2 & 205.6 & 210.8 & 212.8 & 206.8 & 208.4 & 210.5 & 204.1 \\
\hline MVPA (min $\cdot$ day $\left.^{-1}\right)$ & 50.9 & 60.1 & 41.6 & 44.9 & 52.6 & 36.3 & 57.0 & 63.3 & 48.4 \\
\hline Meet PA guideline ${ }^{\mathrm{b}}(\%)$ & 23.1 & 30.0 & 13.4 & 15.9 & 21.4 & 8.9 & 30.5 & 38.3 & 18.6 \\
\hline Sleep $\left(\min \cdot\right.$ day $^{-1}$ ) & 543.2 & 588.1 & 499.3 & 544.6 & 581.7 & 495.9 & 541.9 & 567.3 & 502.8 \\
\hline Meet sleep guideline ${ }^{c}(\%)$ & 27.6 & 41.4 & 8.1 & 32.4 & 51.5 & 7.6 & 22.6 & 31.8 & 8.6 \\
\hline $\begin{array}{l}\text { Valid wear time } \\
\text { (hour.day }{ }^{-1} \text { ) }\end{array}$ & $\begin{array}{l}22.8 \\
(1.0)\end{array}$ & $22.4(0.8)$ & $23.4(0.9)$ & $\begin{array}{l}22.9 \\
(1.0)\end{array}$ & $\begin{array}{l}22.4 \\
(0.8)\end{array}$ & $23.5(0.8)$ & $\begin{array}{l}22.7 \\
(1.0)\end{array}$ & $\begin{array}{l}22.3 \\
(0.8)\end{array}$ & $23.3(0.9)$ \\
\hline $\begin{array}{l}\text { Number of days with valid } \\
\text { wear time }\end{array}$ & $5.9(1.6)$ & $6.1(1.0)$ & $5.6(2.1)$ & $6.1(1.4)$ & $\begin{array}{l}6.2 \\
(1.0)\end{array}$ & $6.0(1.7)$ & $5.6(1.8)$ & $\begin{array}{l}6.0 \\
(1.1)\end{array}$ & $5.1(2.4)$ \\
\hline \multicolumn{10}{|l|}{ Dragon Challenge Scores } \\
\hline Overall score & $\begin{array}{l}32.0 \\
(7.4)\end{array}$ & $31.2(7.4)$ & $33.2(7.4)$ & $\begin{array}{l}30.8 \\
(7.2)\end{array}$ & $\begin{array}{l}30.1 \\
(6.7)\end{array}$ & $31.8(7.7)$ & $\begin{array}{l}33.3 \\
(7.5)\end{array}$ & $\begin{array}{l}32.3 \\
(7.9)\end{array}$ & $34.8(6.7)$ \\
\hline Process score & $9.4(3.2)$ & $9.0(3.1)$ & $9.9(3.2)$ & $8.8(3.2)$ & $\begin{array}{l}8.4 \\
(2.9)\end{array}$ & $9.3(3.5)$ & $9.9(3.1)$ & $\begin{array}{l}9.5 \\
(3.2)\end{array}$ & $10.5(2.9)$ \\
\hline Product score & $\begin{array}{l}10.2 \\
(3.5)\end{array}$ & $10.0(3.5)$ & $10.4(3.6)$ & $9.8(3.6)$ & $\begin{array}{l}9.7 \\
(3.4)\end{array}$ & $10.0(3.8)$ & $\begin{array}{l}10.6 \\
(3.5)\end{array}$ & $\begin{array}{l}10.3 \\
(3.6)\end{array}$ & $10.9(3.3)$ \\
\hline Time score & $\begin{array}{l}12.5 \\
(2.1)\end{array}$ & $12.2(2.2)$ & $12.9(1.8)$ & $\begin{array}{l}12.2 \\
(1.8)\end{array}$ & $\begin{array}{l}12.0 \\
(1.8)\end{array}$ & $12.5(1.8)$ & $\begin{array}{l}12.8 \\
(2.2)\end{array}$ & $\begin{array}{l}12.5 \\
(2.5)\end{array}$ & $13.3(1.8)$ \\
\hline
\end{tabular}




\begin{tabular}{|c|c|c|c|c|c|c|c|c|c|}
\hline Variables & $\begin{array}{l}\text { All } \\
\text { schools }\end{array}$ & $\begin{array}{l}\text { Primary } \\
\text { schools } \\
\text { (All) }\end{array}$ & $\begin{array}{l}\text { Secondary } \\
\text { schools } \\
\text { (All) }\end{array}$ & $\begin{array}{l}\text { All } \\
\text { schools } \\
\text { (Girls) }\end{array}$ & $\begin{array}{l}\text { Primary } \\
\text { schools } \\
\text { (Girls) }\end{array}$ & $\begin{array}{l}\text { Secondary } \\
\text { schools } \\
\text { (Girls) }\end{array}$ & $\begin{array}{l}\text { All } \\
\text { schools } \\
\text { (Boys) }\end{array}$ & $\begin{array}{l}\text { Primary } \\
\text { schools } \\
\text { (Boys) }\end{array}$ & $\begin{array}{l}\text { Secondary } \\
\text { schools } \\
\text { (Boys) }\end{array}$ \\
\hline
\end{tabular}

Note. ${ }^{a}$ Each behavioural time-use set was transformed to compositional means (expressed as the geometric mean of each behaviour, linearly adjusted to collectively sum to 1440 minutes); b Minimum of 60 min. day ${ }^{-1}$ MVPA averaged over the week ${ }^{24}$; ${ }^{c}$ Minimum of 9 hours night $^{-1}$ sleep ${ }^{25}$; Dragon Challenge scores ranges were: overall score $0-54$, process score $0-18$, product score $0-18$, time score 0-18.

Table 2 summarises the results from the regression models and displays the significant associations between the activity composition ILR coordinates and DC scores, stratified by sex and school type (see Supplementary Material 1 for full model outputs).

Table 2

Associations between activity composition and motor competence outcomes

\begin{tabular}{|c|c|c|c|c|c|c|c|c|c|c|c|c|c|c|c|c|}
\hline \multirow{2}{*}{$\begin{array}{l}\text { Motor } \\
\text { Competence } \\
\text { Outcomes }\end{array}$} & \multicolumn{2}{|c|}{ All schools } & \multicolumn{2}{|c|}{$\begin{array}{l}\text { Primary } \\
\text { Schools (All) }\end{array}$} & \multicolumn{2}{|c|}{$\begin{array}{l}\text { Secondary } \\
\text { Schools (All) }\end{array}$} & \multicolumn{2}{|c|}{$\begin{array}{l}\text { All schools } \\
\text { (Girls) }\end{array}$} & \multicolumn{2}{|c|}{$\begin{array}{l}\text { Primary } \\
\text { Schools } \\
\text { (Girls) }\end{array}$} & \multicolumn{2}{|c|}{$\begin{array}{l}\text { Secondary } \\
\text { Schools (Girls) }\end{array}$} & \multicolumn{2}{|c|}{$\begin{array}{l}\text { All schools } \\
\text { (Boys) }\end{array}$} & \multicolumn{2}{|c|}{$\begin{array}{l}\text { Primary } \\
\text { Schools } \\
\text { (Boys) }\end{array}$} \\
\hline & $X^{2}$ & $P$ & $X^{2}$ & $p$ & $X^{2}$ & $p$ & $X^{2}$ & $p$ & $X^{2}$ & $p$ & $X^{2}$ & $p$ & $X^{2}$ & $p$ & $X^{2}$ & $p$ \\
\hline $\begin{array}{l}\text { Overall } \\
\text { score }\end{array}$ & 17.20 & $<0.001$ & 4.21 & 0.24 & 17.59 & $<0.001$ & 13.66 & $<0.001$ & 4.76 & 0.19 & 11.86 & 0.008 & 12.94 & $<0.001$ & 11.69 & 0.0 \\
\hline $\begin{array}{l}\text { Process } \\
\text { score }\end{array}$ & 23.80 & $<0.001$ & 3.74 & 0.29 & 23.00 & $<0.001$ & 15.46 & $<0.001$ & 3.30 & 0.35 & 15.38 & 0.002 & 14.57 & $<0.001$ & 8.72 & 0.0 \\
\hline $\begin{array}{l}\text { Product } \\
\text { score }\end{array}$ & 6.19 & 0.10 & 0.90 & 0.83 & 9.38 & 0.02 & 9.51 & 0.02 & 9.56 & 0.02 & 8.93 & 0.03 & 6.25 & 0.10 & 8.16 & 0.0 \\
\hline Time score & 8.87 & 0.03 & 10.84 & 0.01 & 5.31 & 0.15 & 2.04 & 0.56 & 4.26 & 0.23 & 1.06 & 0.79 & 8.01 & 0.045 & 10.52 & 0.0 \\
\hline
\end{tabular}

Note. Activity composition expressed as isometric log ratios. Models adjusted for age (excluding stratified by school type analysis), sex (excluding stratified $k$ BMI z-score, and IMD decile. Bolded estimated are significant at $p<0.05$ ('school' as the single, random intercept to account for nesting).

Beta coefficients for the first pivot coordinate of the models showing significant associations between activity composition and outcomes are presented in Table 3. 
Table 3

Relationship between motor competence outcomes that were significantly associated with the activity composition, and the activity behaviour isometric logratio regression coefficients and standard error (1 ilr (SE))

\begin{tabular}{|c|c|c|c|c|}
\hline $\begin{array}{l}\text { Motor Competence Outcomes } \\
\text { Dragon Challenge Scores }\end{array}$ & $\beta_{1}$ ilr Sleep & $\beta_{1}$ ilrST & $\beta_{1}$ ilr LPA & $\beta_{1}$ ilr MVPA \\
\hline \multicolumn{5}{|l|}{ All Schools } \\
\hline Overall score & $-2.45(2.25)$ & $-0.60(1.80)$ & $-1.76(2.64)$ & $4.81(1.37)$ \\
\hline Process score & $-1.00(0.96)$ & $-0.52(0.77)$ & $-0.86(1.13)$ & $2.40(0.59)$ \\
\hline Time score & $-0.43(0.64)$ & $-0.03(0.51)$ & $-0.58(0.75)$ & $1.04(0.39)$ \\
\hline \multicolumn{5}{|l|}{ Primary Schools (All) } \\
\hline Time score & $1.52(1.22)$ & $-1.54(1.05)$ & $-1.72(1.04)$ & $1.74(0.64)$ \\
\hline \multicolumn{5}{|l|}{ Secondary Schools (All) } \\
\hline Overall score & $-7.34(2.92)$ & $-0.26(2.22)$ & $3.06(4.21)$ & $4.54(1.87)$ \\
\hline Process score & $-2.63(1.29)$ & $-0.50(0.98)$ & $0.45(1.86)$ & $2.68(0.83)$ \\
\hline Product score & $-3.17(1.52)$ & $0.11(1.15)$ & $1.48(2.19)$ & $1.58(0.97)$ \\
\hline \multicolumn{5}{|l|}{ All Schools (Girls) } \\
\hline Overall score & $-8.33(4.29)$ & $-0.05(3.92)$ & $5.78(3.69)$ & $2.60(1.89)$ \\
\hline Process score & $-3.48(1.87)$ & $0.09(1.70)$ & $1.67(1.62)$ & $1.73(0.83)$ \\
\hline Product score & $-4.72(2.23)$ & $0.75(2.04)$ & $3.39(1.92)$ & $0.57(0.98)$ \\
\hline \multicolumn{5}{|l|}{ Primary Schools (Girls) } \\
\hline Product score & $-6.07(3.43)$ & $1.70(2.82)$ & $7.71(2.53)$ & $-3.34(1.60)$ \\
\hline \multicolumn{5}{|l|}{ Secondary Schools (Girls) } \\
\hline Overall score & $-14.33(6.16)$ & $3.76(6.43)$ & $5.86(7.03)$ & $4.72(2.82)$ \\
\hline Process score & $-5.64(2.75)$ & $0.94(2.87)$ & $1.79(3.14)$ & $2.90(1.26)$ \\
\hline Product score & $-7.69(3.18)$ & $3.11(3.31)$ & $2.71(3.62)$ & $1.86(1.45)$ \\
\hline \multicolumn{5}{|l|}{ All Schools (Boys) } \\
\hline Overall score & $0.56(2.88)$ & $-0.26(2.08)$ & $-7.28(3.72)$ & $6.98(2.02)$ \\
\hline Process score & $0.20(1.19)$ & $-0.34(0.86)$ & $-2.86(1.54)$ & $3.00(0.83)$ \\
\hline Time score & $-0.55(0.88)$ & $0.08(0.64)$ & $-1.19(1.14)$ & $1.67(0.62)$ \\
\hline \multicolumn{5}{|l|}{ Primary Schools (Boys) } \\
\hline Overall score & $9.97(5.52)$ & $-4.65(4.86)$ & $-12.67(5.01)$ & $7.34(3.11)$ \\
\hline Process score & $2.82(2.29)$ & $-0.94(2.00)$ & $-4.81(2.08)$ & $2.93(1.29)$ \\
\hline Product score & $5.59(2.63)$ & $-2.34(2.31)$ & $-5.13(2.38)$ & $1.88(1.48)$ \\
\hline Time score & $1.99(1.75)$ & $-1.63(1.50)$ & $-2.95(1.58)$ & $2.59(0.99)$ \\
\hline \multicolumn{5}{|l|}{ Secondary Schools (Boys) } \\
\hline Process score & $-1.81(1.50)$ & $-0.63(0.97)$ & $0.23(2.37)$ & $2.20(1.15)$ \\
\hline
\end{tabular}

Starting with the average composition of durations for each activity (Table 1) and using compositional isotemporal substitution, Figure 1a-c and Supplementary Materials 2 and 3 present predicted differences in DC scores when incremental durations of time were added/subtracted from the most influential activity behaviour (Table 3), and reallocated from/to one other activity, keeping all remaining activities constant. For all participants, time reallocations to MVPA from any of the other three behaviours was associated with higher overall, process, and time scores (ES ranged from 0.05 to 0.07 ( 5 $\mathrm{min}$ ) and 0.19 to 0.27 (20 min)) (Figure 1a-c and Supplementary Materials 2). Among primary school participants there were positive predicted differences in time score when MVPA replaced any other behaviour ( $E S=0.08(5 \mathrm{~min})$ to $0.30(20 \mathrm{~min})$ ). In secondary school participants, MVPA replacing any other behaviour was associated with positive predicted changes in overall and process scores (ES ranged from 0.08 to 0.10 ( $5 \mathrm{~min}$ ) and 0.28 to 0.35 (20 min)), and negative changes in product score were found when sleep replaced ST, LPA, and MVPA (ES = 0.06 ( 5 min) to 0.29 (20 min)) (Supplementary Materials 2 and 3). 
The sex-stratified by school type compositional isotemporal substitutions showed that (Supplementary Materials 2 and 3 ), for girls, time reallocations to sleep from either ST, LPA, or MVPA was associated with poorer DC scores (ES ranged from 0.03 to 0.10 ( 5 min) and 0.13 to 0.43 (20 min)) and favourable predicted differences in DC scores were found when MVPA or LPA replaced any other activity behaviour (ES ranged from 0.06 to 0.14 (5 min) and 0.23 to 0.63 (20 min)). For boys, replacing sleep, ST, or LPA with MVPA, was associated with positive predicted changes in DC scores (ES ranged from 0.07 to 0.10 ( 5 min) and 0.26 to $0.39(20 \mathrm{~min})$ ) and when LPA replaced sleep, ST, and MVPA unfavourable predicted differences in DC scores (ES ranged from 0.07 to 0.11 (5 min) and 0.31 to 0.49 (20 $\mathrm{min})$ were observed.

\section{Discussion:}

This study is the first to investigate associations between 24-hour activity behaviours and motor competence using a hybrid-based (both process- and productbased outcomes) measure that assesses proficiency in fundamental, combined, and complex movement skills. It adds to previous compositional analysis studies(10-13), by extending to children and adolescents, while stratifying by sex. In all children and adolescents, we found that activity composition was significantly associated with DC overall, process, and time scores. Among primary school participants, activity composition was associated with time score only, while in secondary school participants it was associated with overall, process, and product scores. Overall, relative to other activity behaviours, MVPA had the greatest association with motor competence outcomes. The largest positive predicted differences in motor competence outcomes occurred when MVPA replaced LPA.

Congruent with previous systematic review evidence supporting positive isolated associations between PA levels and motor competence(2, 7$)$, these findings reinforce the key role of MVPA for children's and adolescents' motor competence. They also support previous compositional studies examining associations between activity behaviours and motor competence in preschool and primary school children $(10,11)$. These showed that reallocating time to MVPA, elicited the largest positive predicted change in overall motor competence scores $(10,11)$. However, contrary to our findings, another compositional study found that relocating time to LPA or sleep, at the expense of ST, was associated with positive differences in motor competence in primary school children from a low socioeconomic status area(12). Nevertheless, despite one recent systematic review finding no evidence for an association between PA and motor competence(3), our findings, as well as others $(10,11)$ provide empirical evidence that MVPA, relative to other behaviours is associated with motor competence outcomes.

Among girls, reallocating time to sleep was associated with negative predicted changes, and increasing MVPA or LPA was associated with positive differences in DC scores. For boys, reallocating time spent to MVPA was associated with positive predicted changes, whereas, increasing time in LPA was associated with negative differences in DC scores. Overall, MVPA had the greatest influence on motor competence outcomes in both boys and girls across school type. The largest positive and negative predicted differences in motor competence outcomes occurred, when MVPA replaced LPA or sleep and when LPA or sleep replaced MVPA, respectively. Again, consistent with previous systematic reviews showing various associations between PA and motor competence in boys and girls $(2,7)$, these findings further emphasise the key role of MVPA for both sexes' motor competence. Notably, no other compositional analysis study(1013) has investigated sex-stratified associations between activity composition and motor competence outcomes. Thus, we provide new evidence for the contention that time spent in MVPA (relative to other activities) is positively associated with motor competence outcomes irrespective of sex.

Using a hybrid-based assessment $(1,6)$, our findings represent a holistic view on the association between activity behaviours and motor competence(1). The largest predicted increases in overall, process, and time scores were observed when MVPA replaced LPA or sleep, whilst the largest predicted decreases occurred when LPA replaced MVPA. Further, the largest predicted increases in product score were observed when LPA replaced MVPA, and greatest decreases were when sleep or LPA replaced MVPA. These results contrast with the recent systematic review evidence(3), as well as a non-compositional study using both process- and product-based assessments of motor competence no associations between MVPA and motor competence $(3,26)$. Further, previous research utilising compositional data analysis has only considered motor competence outcomes using a process-based approach(10-13), and whilst positive changes in motor competence were found(10-13), the current study provides further evidence that associations exist between MVPA (relative to other activity behaviours) and both process- and product-based motor competence outcomes. Thus, irrespective of a process- or product-based assessment approach used to assess motor competence, our findings suggest that enabling engagement in MVPA is beneficial for motor competence in all children and adolescents.

Interestingly, the predicted differences in motor competence outcomes were greatest when MVPA was replaced by sleep or LPA, rather than when MVPA replaced these behaviours. These asymmetrical associated differences in physical outcomes involving MVPA have previously been observed in youth studies considering adiposity and fitness(27). Furthermore, some studies have reported negative associations between ST and motor competence(7, 28). We found that ST, relative to the other activity behaviours was not significantly associated with any of the motor competence outcomes. However, in Table 2 , a negative association was present for the full sample, but inconsistent in the sub-group analyses, therefore, the lack of consistent significant association may be a combination of statistical artefact (i.e., small sample sizes) and potential misclassification of LPA as ST. Nonetheless, further studies on the potential negative effects of ST on the development of motor competence are warranted.

In line with the recent review evidence reporting a lack of association between PA and motor competence(3), it is noteworthy, that there were no significant associations observed between the activity compositions and numerous DC scores (Table 2). These non-significant associations between activity behaviours and outcomes requires further investigation, but it may mean that other influencing factors better predict motor competence outcomes( $3,4,8)$. Therefore, children/adolescents may benefit other interventions such as appropriately challenging opportunities, instruction and feedback during activities, to complement increases in MVPA, as opposed to just time reallocated to MVPA(2-5, 8).

Strengths of this study included wrist-worn device-based assessment of 24-hour activity behaviours and use of compositional data analysis to examine how the full activity composition related to motor competence outcomes. Moreover, this is the first compositional analysis study to investigate motor competence outcomes, measured using a hybrid-based assessment that evaluates proficiency in fundamental, combined, and complex movement skills. However, the 
study is not without limitations. The cross-sectional design precludes any claims of causal inferences and directionality between the activity composition and motor competence outcomes. There is also evidence for a motor competence to PA association, thus there is a possibility of reverse causality or bi-directional associations $(2,3,5)$. Furthermore, the authors acknowledge an increased type 2 error risk from conducting a large number of hypothesis tests (as a result of stratification by sex and school type). Nevertheless, the novel findings that MVPA, relative to other behaviours, was associated with various motor competence outcomes in both primary and secondary school participants, suggests that the association between MVPA and motor competence is still present during middle to late childhood and adolescence $(2,3,5)$. We also had an imbalanced sample of primary and secondary school participants, who were relatively homogenous in terms of area-level socioeconomic status, which limits generalisability. Therefore, future studies should extend this work across the full spectrum of area-level socioeconomic status/neighbourhood deprivation. Finally, although the activity behaviours were defined using validated wrist-worn acceleration cut-points for LPA and MVPA, these reflect absolute intensity rather than relative intensity for each participant and therefore, may have resulted in some misclassification of activity behaviours.

\section{Conclusion:}

Overall, the activity behaviour compositions were associated with some but not all motor competence outcomes. Among both sexes, relative to other activity behaviours, MVPA had the greatest associations overall on outcomes. When time was reallocated, the largest positive predicted differences in outcomes occurred when MVPA replaced LPA or sleep. Therefore, our findings reinforce the key role of MVPA for children and adolescents' motor competence and highlight the value of compositional data analysis for understanding the collective influence of activity behaviours on motor competence (using a hybridbased approach), which could guide the focus of motor competence interventions. For example, practical approaches to increase MVPA are advocated (e.g., active classroom breaks, physically active learning, outdoor play, high quality PE, structured sport/exercise, family activities)(29, 30). Future studies should investigate associations between activity behaviours and motor competence in samples that are more representative of key demographics, expanding the generalisability of results within longitudinal designs.

\section{Abbreviations}

PA - physical activity

IMD - Indices of Multiple Deprivation

$\mathrm{BMI}$ - body mass index

ENMO - Euclidean Norm Minus One

LPA - light physical activity

MVPA - moderate-to-vigorous physical activity

ST - sedentary time

DC - Dragon Challenge

ILR - isometric log ratios

ES - effect size

\section{Declarations}

- Ethical approval and consent to participate: Ethics approval was provided by the Edge Hill University Research Ethics Committee (\#SPA-REC-2018-007). All participants provided signed informed parent/carer consent and assent.

- Consent for publication: Not applicable

- Availability of data and materials: The datasets used and/or analysed during the current study are available from the corresponding author on reasonable request.

- Competing interests:The authors declare that they have no conflict of interests. The results are presented clearly, honestly, and without fabrication, falsification, or inappropriate data manipulation.

- Funding: The current study was funded by The Waterloo Foundation [grant \#1669-3509]. The Waterloo Foundation had no role in the design of the study, the collection, analysis, and interpretation of the data, and in the writing of the manuscript.

- Authors' contributions: RT designed and managed the study and collected, processed, cleaned the data, performed exploratory data analysis, and led the writing of the manuscript. SJF designed the study, obtained funding, and performed exploratory data analysis. JRD performed the main data analysis. DD advised and contributed to the data analysis and interpretation. AJA contributed to the study design and protocol. All authors contributed to writing the manuscript, and read and approved the final manuscript version.

- Acknowledgements: The authors gratefully acknowledge the role of West Lancashire Sport Partnership and the participating schools, including teachers, the children/adolescents and their parents/guardians, for their engagement in the study.

\section{References}


1. Hulteen RM, Barnett LM, True L, Lander NJ, del Pozo Cruz B, Lonsdale C. Validity and reliability evidence for motor competence assessments in children and adolescents: A systematic review. Journal Sports Sciences. 2020;38(15):1717-98.

2. Robinson LE, Stodden DF, Barnett LM, Lopes VP, Logan SW, Rodrigues LP, et al. Motor Competence and its Effect on Positive Developmental Trajectories of Health. Sports Medicine. 2015;45(9):1273-84.

3. Barnett LM, Webster EK, Hulteen RM, de Meester A, Valentini NC, Lenoir M, et al. Through the Looking Glass: A Systematic Review of Longitudinal Evidence, Providing New Insight for Motor Competence and Health. Sports Medicine [Internet]. 2021;1-46. Available from:

https://link.springer.com/10.1007/s40279-021-01516-8

4. Tyler R, Mackintosh KA, Foweather L, Edwards LC, Stratton G. Youth motor competence promotion model: a quantitative investigation into modifiable factors. Journal of Science and Medicine in Sport. 2020;23(10):955-61.

5. Stodden DF, Goodway JD, Langendorfer SJ, Roberton MA, Rudisill ME, Garcia C, et al. A developmental perspective on the role of motor skill competence in physical activity: An emergent relationship. Quest. 2008;60(2):290-306.

6. Tyler R, Foweather L, Mackintosh KA, Stratton G. A dynamic assessment of children's physical competence: The dragon challenge. Medicine and Science in Sports and Exercise. 2018;50(12):2474-87.

7. Barnett LM, Lai SK, Veldman SLC, Hardy LL, Cliff DP, Morgan PJ, et al. Correlates of Gross Motor Competence in Children and Adolescents: A Systematic Review and Meta-Analysis. Sports Medicine. 2016;46(11):1663-88.

8. Hulteen RM, Morgan PJ, Barnett LM, Stodden DF, Lubans DR. Development of Foundational Movement Skills: A Conceptual Model for Physical Activity Across the Lifespan. Sports Medicine. 2018;48(7):1533-40.

9. Dumuid D, Pedišić Ž, Stanford TE, Martín-Fernández JA, Hron K, Maher CA, et al. The compositional isotemporal substitution model: A method for estimating changes in a health outcome for reallocation of time between sleep, physical activity and sedentary behaviour. Statistical Methods in Medical Research. 2019;28(3):846-57.

10. Burns RD, Kim Y, Byun W, Brusseau TA. Associations of school day sedentary behavior and physical activity with gross motor skills: Use of compositional data analysis. Journal of Physical Activity and Health. 2019;16(10):811-7.

11. Mota JG, Clark CCT, Bezerra TA, Lemos L, Reuter CP, Mota JAPS, et al. Twenty-four-hour movement behaviours and fundamental movement skills in preschool children: A compositional and isotemporal substitution analysis. Journal of Sports Sciences. 2020;38(18):2071-9.

12. Smith E, Fazeli F, Wilkinson K, Clark CCT. Physical behaviors and fundamental movement skills in British and Iranian children: An isotemporal substitution analysis. Scandinavian Journal of Medicine and Science in Sports. 2021;31(2):398-404.

13. Martins CM de L, Clark CCT, Tassitano RM, Filho AN de S, Gaya AR, Duncan MJ. School-Time Movement Behaviors and Fundamental Movement Skills in Preschoolers: An Isotemporal Reallocation Analysis. Perceptual and Motor Skills. 2021;128(4):1317-36.

14. Fairclough SJ, Tyler R, Dainty JR, Dumuid D, Richardson C, Shepstone L, et al. Cross-sectional associations between 24-hour activity behaviours and mental health indicators in children and adolescents: A compositional data analysis. Journal of Sports Sciences. 2021;39(14):1602-14.

15. Ministry of Housing Communities \& Local Government. English Indices of Deprivation 2019 [Internet]. 2019 [cited 2021 Sep 17]. Available from: https://imd-by-postcode.opendatacommunities.org/imd/2019

16. Cole TJ, Bellizzi MC, Flegal KM, Dietz WH. Establishing a standard definition for child overweight and obesity worldwide: international survey. BMJ. 2000;320:1-6.

17. Migueles JH, Rowlands A v., Huber F, Sabia S, van Hees VT. GGIR: A Research Community-Driven Open Source R Package for Generating Physical Activity and Sleep Outcomes From Multi-Day Raw Accelerometer Data. Journal for the Measurement of Physical Behaviour. 2019;2(3):188-96.

18. van Hees VT, Gorzelniak L, Dean León EC, Eder M, Pias M, Taherian S, et al. Separating Movement and Gravity Components in an Acceleration Signal and Implications for the Assessment of Human Daily Physical Activity. PLoS ONE. 2013;8(4):1-10.

19. van Hees VT, Fang Z, Langford J, Assah F, Mohammad A, M da Silva IC, et al. Autocalibration of accelerometer data for free-living physical activity assessment using local gravity and temperature: an evaluation on four continents. J Appl Physiol [Internet]. 2014;117:738-44. Available from: http://www.jappl.org

20. Hildebrand M, van Hees VT, Hansen BH, Ekelund U. Age group comparability of raw accelerometer output from wrist-and hip-worn monitors. Medicine and Science in Sports and Exercise. 2014;46(9):1816-24.

21. Hurter L, Fairclough S, Knowles Z, Porcellato L, Cooper-Ryan A, Boddy L. Establishing Raw Acceleration Thresholds to Classify Sedentary and Stationary Behaviour in Children. Children. 2018;5(172):1-18.

22. van Hees VT, Sabia S, Jones SE, Wood AR, Anderson KN, Kivimäki M, et al. Estimating sleep parameters using an accelerometer without sleep diary. Scientific Reports. 2018;8(1):1-11.

23. Dumuid D, Maher C, Lewis LK, Stanford TE, Martín Fernández JA, Ratcliffe J, et al. Human development index, children's health-related quality of life and movement behaviors: a compositional data analysis. Quality of Life Research. 2018;27(6):1473-82.

24. Department of Health \& Social Care. UK Chief Medical Officers' Physical Activity Guidelines. London: DHSC; 2019.

25. Paruthi S, Brooks LJ, D’Ambrosio C, Hall WA, Kotagal S, Lloyd RM, et al. Recommended amount of sleep for pediatric populations: A consensus statement of the American Academy of Sleep Medicine. Journal of Clinical Sleep Medicine. 2016;12(6):785-6.

26. Britton, Belton S, Issartel J. Small fish, big pond: The role of health-related fitness and perceived athletic competence in mediating the physical activitymotor competence relationship during the transition from primary to secondary school. Journal of Sports Sciences. 2019;37(22):2538-48.

27. Fairclough SJ, Dumuid D, Taylor S, Curry W, McGrane B, Stratton G, et al. Fitness, fatness and the reallocation of time between children's daily movement behaviours: An analysis of compositional data. International Journal of Behavioral Nutrition and Physical Activity. 2017;14(64):1-12. 
28. Adank AM, van Kann DHH, Hoeboer JJAA, de Vries SI, Kremers SPJ, Vos SB. Investigating motor competence in association with sedentary behavior and physical activity in 7-to 11-year-old children. International Journal of Environmental Research and Public Health. 2018;15(2470):1-11.

29. Daly-Smith A, Quarmby T, Archbold VSJ, Corrigan N, Wilson D, Resaland GK, et al. Using a multi-stakeholder experience-based design process to codevelop the Creating Active Schools Framework. International Journal of Behavioral Nutrition and Physical Activity. 2020;17(13):1-12.

30. Rhodes RE, Guerrero MD, Vanderloo LM, Barbeau K, Barbeau K, Birken CS, et al. Development of a consensus statement on the role of the family in the physical activity, sedentary, and sleep behaviours of children and youth. Vol. 17, International Journal of Behavioral Nutrition and Physical Activity. BioMed Central Ltd.; 2020. p. 1-31.

\section{Figures}
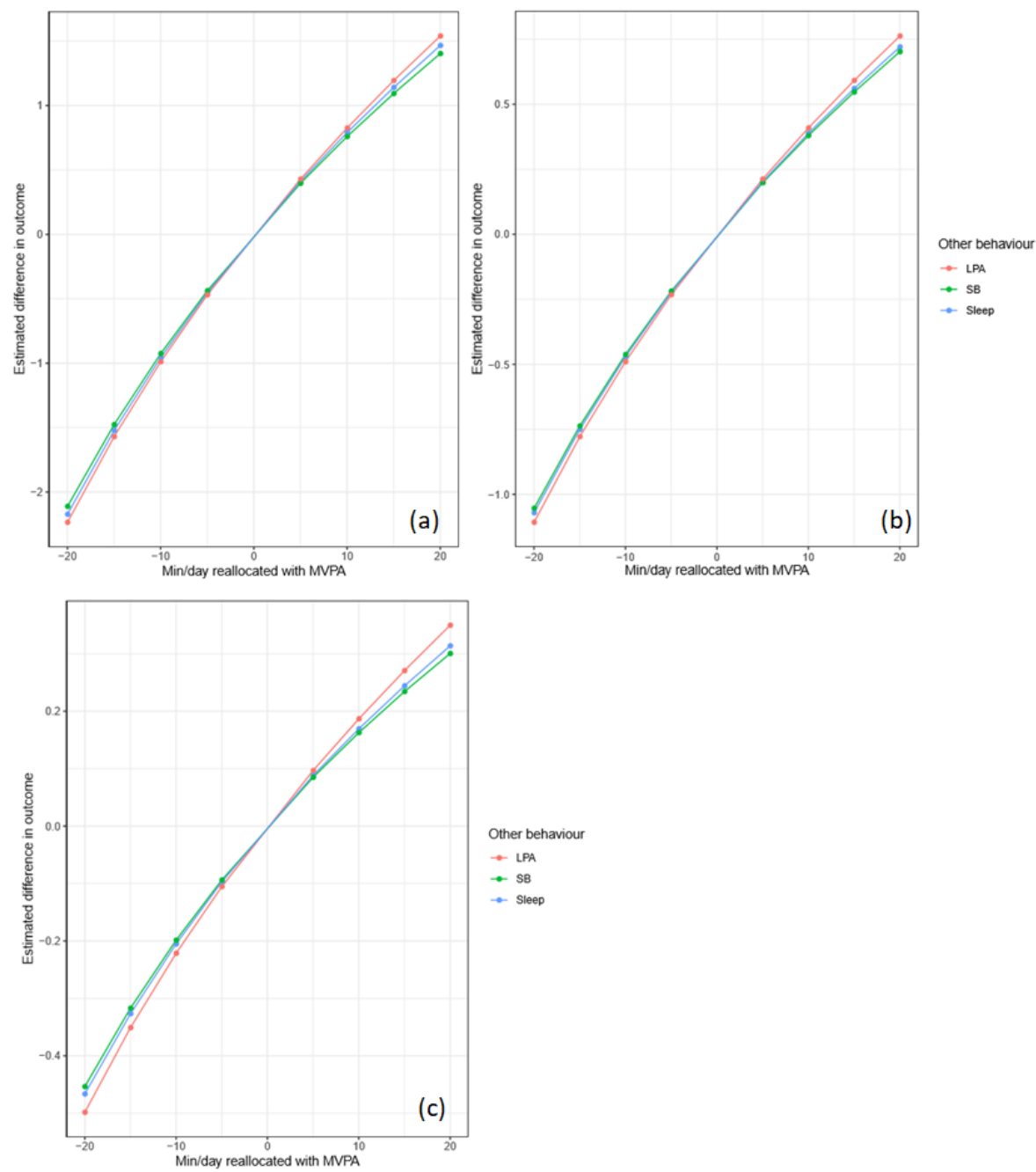

\section{Figure 1}

1a, 1b and 1c.Estimated difference in Dragon Challenge scores associated with time reallocation between pairs of behaviours: difference in all participants' (a) overall, (b) process and (c) time scores associated with the difference in MVPA to each of the remaining behaviours. For example, adding 20 minutes to MVPA at the expense of LPA (red line) while keeping sleep and ST constant was associated with a predicted increase in overall score of 1.54 units.

\section{Supplementary Files}

This is a list of supplementary files associated with this preprint. Click to download.

- SupplementaryMaterial1ILRRegressionModels.docx

- SupplementaryMaterial2TimeReallocations.docx

- SupplementaryMaterial30neForOneTimeReallocations.docx

- SupplementaryMaterial4VariationMatrices.docx 
- SupplementaryMaterial5STROBEchecklist.docx

Page $11 / 11$ 